\title{
In response to "Lipomatosis of the sciatic nerve secondary to compression by a desmoid tumor"
}

\author{
Blake D. Niederhauser • Robert J. Spinner • \\ B. Matthew Howe • Kimberly K. Amrami
}

Received: 11 October 2013 / Accepted: 14 October 2013/Published online: 26 November 2013

(C) ISS 2013

Dear Editor,

We read with great interest the case report entitled "Lipomatosis of the sciatic nerve secondary to compression by a desmoid tumor." We respectfully disagree with the authors' interpretation of the imaging and their conclusions.

Our group has had a particular interest in the clinical and radiological features of patients with lipomatosis of nerve (LN) and neuromuscular choristoma (NMC). The images provided in this report are strikingly similar to those published in our recent publication "Neuromuscular choristoma: characteristic magnetic resonance imaging findings and association with postbiopsy fibromatosis" [1], which included only biopsy-proven examples of NMC and fibromatosis (desmoid tumor). While the authors believe that the radiological features in their case are pathognomonic for LN, including the "coaxial cable sign" $[2,3]$, we feel that the imaging characteristics of the abnormal sciatic nerve in this report, namely, the non-enhancing fusiform enlargement of a large proximal peripheral nerve, and a $<50 \%$ fatty component, are more diagnostic of NMC than LN. Indeed, the sciatic nerve is the most common site for NMC, a rare one for LN, and this case overall is an excellent appearance of NMC complicated by aggressive fibromatosis.

The postulated new association of $\mathrm{LN}$ and a desmoid tumor described in this case report is presumptive. While excellent radiological-pathological correlation between the enhancing mass component and the desmoid tumor is noted on biopsy, there is no tissue diagnosis of the sciatic nerve lesion itself. Nor would we have recommended a biopsy of it, owing to the potential exacerbation of the desmoid following surgery or biopsy of NMC $[1,4,5]$. Instead, we would try to support the diagnosis of the different clinical phenotypes of NMC and LN (NMC is associated with nerve territory undergrowth and LN with nerve territory overgrowth) [1]. We believe that the presented case offers additional evidence for the curious and unexplained association of NMC with desmoid development.

\section{References}

1. Niederhauser BD, Spinner RJ, Jentoft ME, Everist BM, Matsumoto JM, Amrami KK. Neuromuscular choristoma: characteristic magnetic resonance imaging findings and association with post-biopsy fibromatosis. Skeletal Radiol. 2013;42(4):567-77.

2. De Maeseneer M, Jaovisidha S, Lenchik L, et al. Fibrolipomatous hamartoma: MR imaging findings. Skeletal Radiol. 1997;26(3):155-60.

3. Marom EM, Helms CA. Fibrolipomatous hamartoma: pathognomonic on MR imaging. Skeletal Radiol. 1999;28(5):260-4.

4. Hebert-Blouin MN, Scheithauer BW, Amrami KK, Durham SR, Spinner RJ. Fibromatosis: a potential sequela of neuromuscular choristoma. J Neurosurg. 2012;116(2):399-408.

5. Hebert-Blouin MN, Amrami KK, Spinner RJ. Letter to the editor: addendum: evidence supports a "no-touch" approach to neuromuscular choristoma. J Neurosurg. 2013. doi:10.3171/2013.3.JNS13444.
B. D. Niederhauser • B. M. Howe · K. K. Amrami

Department of Radiology, Mayo Clinic, Rochester, MN, USA

R. J. Spinner $(\bowtie) \cdot$ K. K. Amrami

Department of Neurosurgery, Mayo Clinic, Rochester, MN, USA

e-mail: Spinner.Robert@mayo.edu

K. K. Amrami

W2 Radiology, 200 1st. St SW, Rochester, MN 55905, USA 